\title{
Two-degree-of-freedom powered prosthetic wrist
}

\author{
Peter J. Kyberd, PhD; ${ }^{1 *}$ Edward D. Lemaire, PhD; ${ }^{2}$ Erik Scheme, MSc; ${ }^{1}$ Catherine MacPhail, BSc; ${ }^{1}$ Louis \\ Goudreau, BASc, PEng; ${ }^{2}$ Greg Bush, BA, CP(c); ${ }^{1}$ Marcus Brookshaw, BSc ${ }^{1}$ \\ ${ }^{1}$ Institute of Biomedical Engineering, University of New Brunswick, Fredericton, Canada; ${ }^{2}$ The Ottawa Hospital Reha- \\ bilitation Centre, Ottawa, Canada
}

\begin{abstract}
Prosthetic wrists need to be compact. By minimizing space requirements, a wrist unit can be made for people with long residual limbs. This prosthetic wrist uses two motors arranged across the arm within the envelope of the hand. The drive is transmitted by a differential so that it produces wrist flexion and extension, pronation and supination, or a combination of both. As a case study, it was controlled by a single-prosthesis user with pattern recognition of the myoelectric signals from the forearm. The result is a compact, two-degree-of-freedom prosthetic wrist that has the potential to improve the functionality of any prosthetic hand by creating a hand orientation that more closely matches grasp requirements.
\end{abstract}

Key words: control, Controller-Area Network bus, distributed control, electromyographic control, electromyography, graphical user interface, microprocessor control, myoelectric control, pattern recognition, powered wrist, prosthetic wrist, twofunction wrist, upper-limb loss, upper-limb prosthetics.

\section{INTRODUCTION}

The constraints placed on the design of prosthetic wrists used for powered hands typically limit the degrees of freedom (DOFs) to a single axis. This DOF is usually pronation and supination (forearm rotation), whether the design is passive or driven. Increasingly, designs with extra passive motions are being introduced. While devices based on a ball joint such as the Omni Wrist (formerly VASI Omni Wrist, it is now known as the Myolino Wrist 2000 [Otto Bock HealthCare; Duderstadt, Germany]), have provided enhanced function for children, similar ball joints are available for the adult population from Otto Bock HealthCare, VASI, and Texas Assistive Devices, LLC (Brazoria, Texas). These multiple-DOF wrists can improve hand positioning, reduce the need for compensatory arm motions to position the hand, and consequently, make activities easier to perform [1-2]. More recently, Motion Control, Inc (Salt Lake City, Utah) [3] and Otto Bock HealthCare launched new passive wristflexion designs for adults that could change the way users perform activities. However, powered flexion, while promised by manufacturers, has so far been limited to the combined action in the Centri Hand (Centri AB; Sollentuna, Sweden). Only the Sven Hand [4-5], its successor, the ES Hand [6], and the commercial Shanghai Kesheng hands (Shanghai Kesheng Prostheses Co, Ltd; Shanghai, China) are able to power wrist flexion and extension or pronation and supination independently.

This article describes a design for a two-DOF wrist based around a differential mechanism that combines both wrist flexion and extension and pronation and supination

Abbreviations: $\mathrm{ACE}=$ Acquisition and Control Environment, CAN-bus $=$ Controller-Area Network bus, $\mathrm{DOF}=$ degree of freedom, EMG = electromyography, ToMPAW = Totally Modular Prosthetic Arm with High Workability.

*Address all correspondence to Peter J. Kyberd, PhD; Institute of Biomedical Engineering, University of New Brunswick, 25 Dineen Dr, PO Box 4400, Fredericton, NB E3B 5A3, Canada; 506-458-7025; fax: 506-453-4827.

Email: pkyberd@unb.ca

DOI:10.1682/JRRD.2010.07.0137 
in a format aiming for practical use in prosthetic applications. The design uses two motors within the palm placed parallel to the anatomical wrist. Both motors drive both DOFs through the differential mechanism. By using two motors synergistically, two DOFs can be driven with close to twice the torque provided using two separate motors.

\section{BACKGROUND}

The differential is an effective mechanism that has many applications, from vehicle drivetrains to mechanical calculation. Mechanical differentials can be used to split one rotating shaft into two motions or to combine two motions into one output. While its use in vehicle drivetrains is common, differentials can be used for many other applications, such as changing output motions when one or both of the output shafts are braked, thereby changing the axis of rotation. For example, Porter and Lesley used a mechanical differential in an elegant wheelchair design [7]. Conventional self-propelled wheelchairs require a hand on each wheel rim to move in a straight line. When a user has only one nondisabled upper limb, both wheels cannot be easily driven. A second wheel rim on the nondisabled side of the wheelchair allows both wheels to be driven by the user, but this approach requires great strength and large hands to hold both wheel rims to propel the chair efficiently. It also requires dexterity to drive the wheels in opposite directions to turn the wheelchair. The drive was changed by Porter and Lesley by passing the motion through a differential. When the shafts are free, both wheels are driven forward by a single wheel rim. However, when a brake is applied to one side of the differential, the resulting output drives the wheels in opposite directions, enabling singlehanded operation to rotate the wheelchair on the spot. This principle is similar to the shift in axis used in this prosthetic wrist design.

\section{THE WRIST: NATURAL AND PROSTHETIC}

To be practical, a prosthetic arm must find a balance between dexterity, space, and weight. In addition, the number of active axes is typically reduced to a manageable number to make the device easy to use, thereby reducing the number of available motions. Users are very adept at compensating for the missing DOFs by changing the motions of their arms and body. Compensatory motions are easy to achieve, requiring the body and residual limb to use a wider range of irregular motions compared with nondisabled persons [1-2]. The resulting poor biomechanics often put greater forces on the anatomy and require greater ranges of motion and repetitive motions, all of which are associated with overuse injuries [8]. Such injuries have been observed in the later lives of prosthesis users [9]. To avoid these potential injuries, more capable and easily used prostheses must be designed by engineers for a user with limb loss and new prosthesis users must be trained by occupational therapists to employ their devices effectively.

The human wrist can flex and extend or abduct and adduct the hand. A third motion, pronation and supination, is actually a function of the forearm provided by the radius and ulna but is commonly regarded as a function of the wrist. When the same function is replaced in a below-elbow prosthesis, pronation and supination are provided as part of the prosthesis because the prosthetic socket tends to restrict rotation of the residual limb. This limitation can be overcome by users by raising the elbow and using humeral elevation to create forearm rotation, thereby using a much larger shoulder range of motion than would otherwise be employed [2]. Since a loss of the forearm tends to limit a person's capability to rotate the hand about the limb's longitudinal axis, a distal prosthetic replacement should have axial rotation built in to reduce the likelihood of long-term injury.

Given the restrictions imposed by long residual-limb lengths and large variations in level of amputation between potential users, building a prosthesis that occupies much of the forearm to drive the hand or wrist is impractical. A device such as the Proto 2 Arm from the U.S. Government-funded Defense Advanced Research Projects Agency prosthetics program [10] that requires the entire forearm would restrict the number of consumers for this device. Therefore, the joint mechanism should occupy as little of the arm proximal to the joint as possible. Likewise, the drive mechanisms for a prosthetic hand ideally must reside within the hand itself and not in the forearm. This is also true for the three rotations of the wrist complex. These constraints, coupled with the need for low weight, have often precluded powered wrist replacements; therefore, the majority of prosthetic wrists have historically been unpowered. When a powered wrist is used, it generally provides only pronation and supination. 
Recent technological advances have benefited the prosthetics field, yielding cheaper and lighter motors, smaller and more powerful microprocessors, and more compact batteries with high stored energy-to-mass ratios. This has enabled newer, more complex solutions, some of which are now applied in the field. For example, the Touch Bionics Hand (Touch Bionics; Livingston, United Kingdom) [11] possesses individually powered fingers. This system was originally designed for users with partial hand loss; hence, users would have an intact wrist to move the hand [12]. Similarly, other manufacturers have also marketed shorter hands that would fit a person with a loss across the carpels (i.e., transcarpal hands from Otto Bock HealthCare and Motion Control, Inc, or powered fingers designed by Vincent Systems GmgH [Weingarten, Germany] [13]). Though designed for people with only partial hand loss, these designs provide space within the hand to fit additional actuators, thereby providing space for users with above-wrist amputation.

The designs described in this article were conceived to allow for two independent motions driven from within a transcarpal-type hand. The wrist unit can be fitted within the hand volume, thereby allowing users with a very long residual limb forearm to use a powered wrist. While most commercial wrists have motion about the pronation and supination axis, there is little evidence of increased functional effectiveness created by choosing this axis. It is probable that the pronation and supination axis is popular because it is the easiest to implement in a robust and simple joint. Only two commercial wrists have powered wrist flexion: the Centri Hand and wrist combination (Centri $\mathrm{AB}$ ) that causes the wrist to flex as the fingers close and the hand and wrist supplied by Shanghai Kesheng Prostheses Co, Ltd, that have separate wrist flexion from hand closure.

While the Centri Hand has limited motions, it originated from the Sven Hand project in the 1970s, which created a three-axis hand and arm system controlled by pattern recognition of the muscle signals in the forearm [4-5]. The result was an elegant design used by a number of persons in the laboratory. Its successor, the ES Hand [6], saw limited field use. The Sven Hand project's most significant contribution was that it showed anecdotally that if a hand with a single DOF is mounted on a multipleaxis wrist and is easily controlled, the hand could be used effectively with fewer compensatory motions than a similar hand without a mobile wrist. More recently, research has shown that wrists with impairment signifi- cantly affect a user's functional range [1-2,14-16]. Therefore, creating a wrist with a greater range of motion that is easier to control is a worthwhile goal.

This article describes a prosthetic wrist design originally conceived as part of the European Union-sponsored Totally Modular Prosthetic Arm with High Workability (ToMPAW) Project (project number DE420), which produced the first modular prosthetic arm system [17]. The ToMPAW consortium brought together engineers from three major prosthetic research projects in Europe: Sven Hand [4], Edinburgh Arm [12], and Southampton Hand [18]. The ToMPAW consortium built on their experiences and expertise to create a modular arm that was the first prosthesis in the field to use a bus-based, microprocessorcontroller architecture [19]. A design concept developed for a two-DOF prosthetic wrist using a differential mechanism that took up as little forearm space as possible was among the outcomes of the ToMPAW Project. Basic designs investigated the concept at the time, but the design was not taken any further. The wrist described here builds on the ToMPAW Project concepts.

Recently, a number of other designs have also been described [20-22], each attempting to add DOFs and thereby improve function. However, these designs have employed the more conventional concepts of one motor per motion.

\section{DESIGN}

A mechanism was designed to provide two wrist motions, pronation and supination and flexion and extension. Two motors were arranged in parallel, oriented in opposite directions and aligned across the base of the hand. Each drive passes through an intermediate gear on the medial and lateral ends of the wrist to shafts running below the motors (Figure 1). The motors drive opposite ends of a differential, with the central wheel attached to the prosthetic socket. When both motors run in opposite directions, the gearing and differential cause the wrist to rotate about the axis through the center of rotation along the long axis of the arm, creating the pronation and supination axis.

A second axis, the flexion and extension axis, is located along the line of the differential. When the two motors turn in the same direction, the drives oppose each other across the differential. This halts forearm rotation and creates a torque around the common shaft, causing 

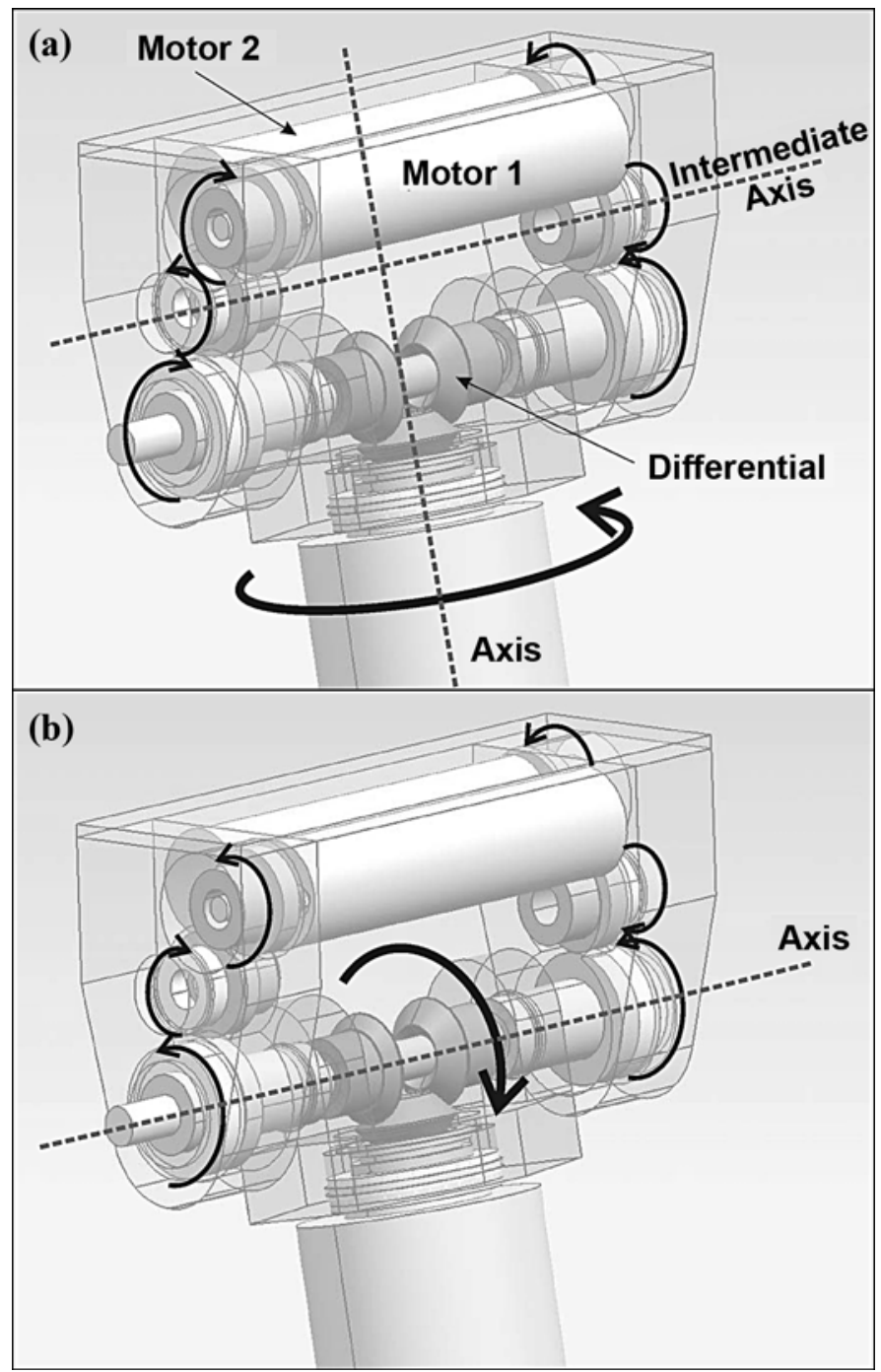

Figure 1.

Wrist actions. (a) Pronation and supination occur when both motors turn in same direction so that two sides of differential rotate in opposite directions. (b) Flexion and extension occur when two motors oppose each other across differential and torque is set up to flex joint.

the wrist to flex or extend (Figure 2). If the motors run at different speeds, the output is a combination of the two axes in proportion to the sum (pronation) and difference (flexion) of the drive speeds. Thus, the combined motion depends on the ratio of the speeds of the two motors. With a differential as the joint axis, the same physical space can be used to produce both wrist rotations. In addition, the combined drives allow for greater torque in any direction when the two motors are combined compared with single-motor designs. Otherwise, for two sepa-

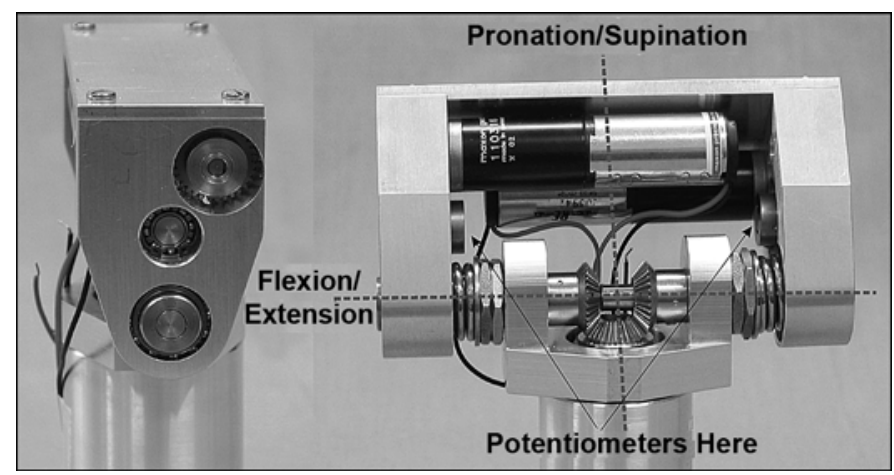

Figure 2.

First-generation wrist design with axes indicated. Joint positions are inferred from readings of potentiometers placed on middle gear (potentiometer locations indicated in image).

rate motors to provide the equivalent torque (one for each axis), larger motors would be required. By orienting the motors across the hand, the mechanism occupies minimal space.

\section{CONTROLLER}

The relative motions of the flexion and extension and pronation and supination axes were measured using potentiometers on the intermediate drives between the outputs of the motor and gearbox combination and the differential. Although the mechanical design can rotate continuously, this creates an unnecessary complication since the natural wrist only provides approximately $100^{\circ}$ of rotation. A greater range of motion would not be functional and could make the device more obvious to the casual observer. Therefore, wrist motion is mechanically and electronically limited to the physiological range.

The wrist is controlled using a microcontroller (model 18F454, Microchip Technology, Inc; Chandler, Arizona) able to drive the two motions. The microcontroller measures position information and controls wrist angle using the relative motions of the motors. The potentiometers measure the position of the motors, and the joint angles are calculated from the sum and difference of the measured angles.

The controller uses serial communications through a Controller-Area Network bus (CAN-bus) [23] to communicate with the rest of the prosthetic arm. A controller node proximal to the wrist can use input signals from switches or conventional myoelectric signals, with the 
user switching between different axes of the wrist and hand and controlling them sequentially. Alternatively, a microprocessor can also be configured as the input processor. The processor can apply pattern-recognition techniques on the signals from multiple muscles on the user's arm, allowing both DOFs to be controlled independently. To the user, the control appears similar to the Sven Hand [4,24-25], with each axis controlled separately by a different command. However, the mathematical processes are very different. Similarly, a microcontroller node can be used to link the CAN-bus with any other serial communications system.

The initial design was produced to test the concept of the two-motor drive with pattern-recognition control. More recently, a smaller, more compact device has also been produced using smaller motor and gearbox combinations [26]. This device is controlled in the same manner as the design described earlier.

\section{APPLICATION: CASE STUDY}

The aim of this initial trial was to test the wrist with a single user employing pattern-recognition techniques from signals in the forearm. This has rarely been possible before. Generally, pattern recognition has been tested with virtual reality simulations of an arm [27-29]. This design represented one of the first opportunities to combine modern pattern-recognition techniques with a complete physical prosthesis. By using a wrist with two motions, the effect of a real device responding to a user's commands could be observed. It also allows for exploration of the new prosthetics techniques needed to provide a stable and repeatable environment for multichannel pattern recognition.

A 22-year-old male with a midlevel transradial amputation was recruited from the Fredericton Limb Clinic (Fredericton, Canada). He used a conventional supracondylar suspension socket and a standard myoelectric hand with two proportional inputs. He was fitted with a new socket embedded with four pairs of dome electrodes distributed around the residual limb, with each pair aligned along the long axis of the limb. The spacing was chosen so that no electrodes were placed over the anterior loading surface of the socket and forearm, thereby minimizing the motion artifact in the acquired signal. Six bipolar amplifiers acquired signals: four amplifiers coinciding with the electrode pairs and two amplifiers bridging diagonally between the pairs on either side of the arm. This setup allowed for more electromyography (EMG) data channels, while minimizing the required electrode contact area and providing a stronger and more comfortable socket fit. The compact wrist design was placed on the end of the socket, with a transcarpal hand (Motion Control, Inc) attached distally (Figure 3).

The University of New Brunswick's Acquisition and Control Environment (ACE) software [30] was modified to provide real-time control outputs to the wrist controller through serial communication. A custom translator board then routed the serial commands to the wrist communication bus. The system was trained to recognize the seven

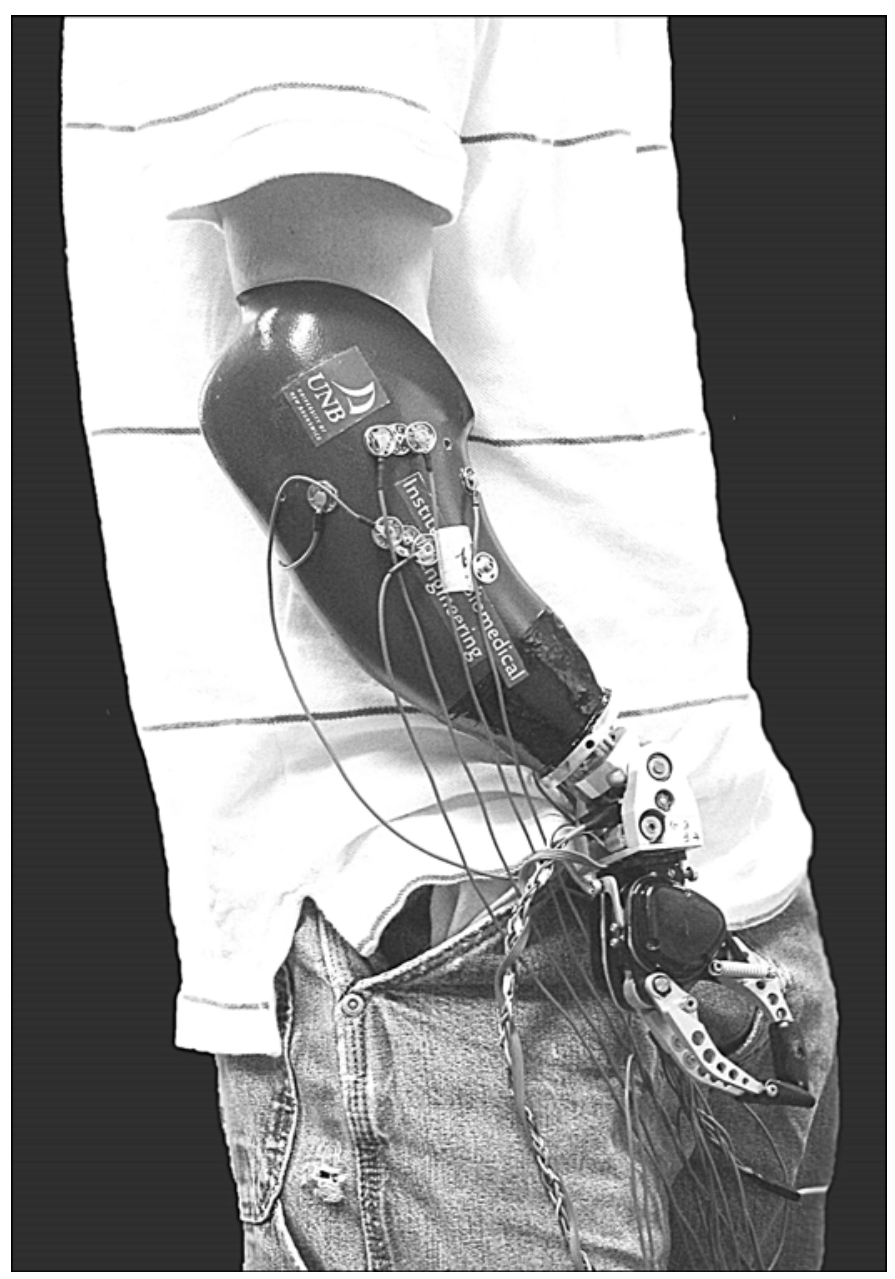

Figure 3.

Experimental configuration for testing two-axis wrist with patternrecognition software. Six channels of electromyographic data from dome electrodes mounted in socket were used to control wrist flexion and extension, wrist pronation and supination, and hand opening and closing. 
types of contractions needed to drive the three DOFs of the wrist and hand (wrist flexion and extension, pronation and supination, hand open and closed, and no movement).

Pattern recognition makes use of spatial and temporal patterns in the EMG data collected from multiple channels. As such, the electrodes do not need to be directly over any specific muscles nor does the user need to elicit any specific contractions. As long as repeatable and discernible contractions are generated, the outputs can be mapped as desired. The simplest and most intuitive arrangement is therefore to use physiologically appropriate contractions that are then mapped to the equivalent prosthetic motion. This system was able to recognize seven motion classes: wrist flexion and extension, pronation and supination, hand open and closed, and no movement. The classifier was used to drive the axes sequentially.

Using the ACE software, the user was given time to practice using the real-time control scheme through a virtual interface with visual feedback [30]. This included asking him to perform the task with both his phantom and contralateral hand to make task visualization easier. Motor velocity commands were derived proportionally to the level of muscle activation in a manner similar to the way conventional EMG signals control velocity.

\section{RESULTS}

Using two motors to drive both motions means that power from both motors is available for single-DOF motions and is distributed between the motions when used simultaneously. Practically, this is sufficient since most prostheses are used in nondominant or support roles [31]. A wrist is generally used to preposition the hand, so speed and flexibility are more important than driven power. The design occupies $32 \mathrm{~mm}$ of arm length within the hand shell, but only $16 \mathrm{~mm}$ on the proximal side of the joint axis, ensuring compatibility with even a long residual limb (Figure 2).

Under coordinated control from the microprocessor, the peak angular velocities for the first prototype wrist were pronation and supination $\left(250{ }^{\circ} \mathrm{S}^{-1}\right)$, flexion and extension $\left(250^{\circ} \mathrm{s}^{-1}\right)$, constant angular velocity pronation and supination $\left(175^{\circ} \mathrm{S}^{-1}\right)$, and constant angular velocity flexion and extension $\left(150{ }^{\circ} \mathrm{s}^{-1}\right)$. At $7 \mathrm{~V}$ and $500 \mathrm{~mA}$, the wrist can generate a $0.073 \mathrm{Nm}$ flexion torque.
The second, smaller version of the wrist created a system $>160 \mathrm{~mm}$ from fingertips to the line of the prosupination axis when attached to the Motion Control Hand (Motion Control, Inc), measured in a similar manner to conventional measurements (Figure 4). For this second phase, the pattern-recognition-based control was evaluated with a prosthesis capable of prepositioning the hand. Overall dimension of the initial design was $96 \mathrm{~mm}$ laterally, and the second design lateral-medial dimension was reduced to $60 \mathrm{~mm}$, while both were $30 \mathrm{~mm}$ anteriorly and $50 \mathrm{~mm}$ distally.

\section{USER APPLICATION}

After a brief familiarization period, the user was asked to perform contractions associated with the seven classes. The data obtained during six contractions and the period of no movement were used to train the classification system, which resulted in accuracies of $>95$ percent for all seven motion classes. The entire process of pattern recognition and controller calibration took only a few minutes and was found to be repeatable from trial to trial. On completion, the user was able to elicit the seven contractions repeatedly and in desired sequences. Subsequently, the user was given time to practice using the real-time control scheme through a virtual interface with visual feedback provided through the ACE software. Once he was satisfied with control in the virtual environment, the physical wrist and hand were enabled. Qualitatively, accurate and usable control of all three DOFs was reported by the user. The correct pattern was clearly able

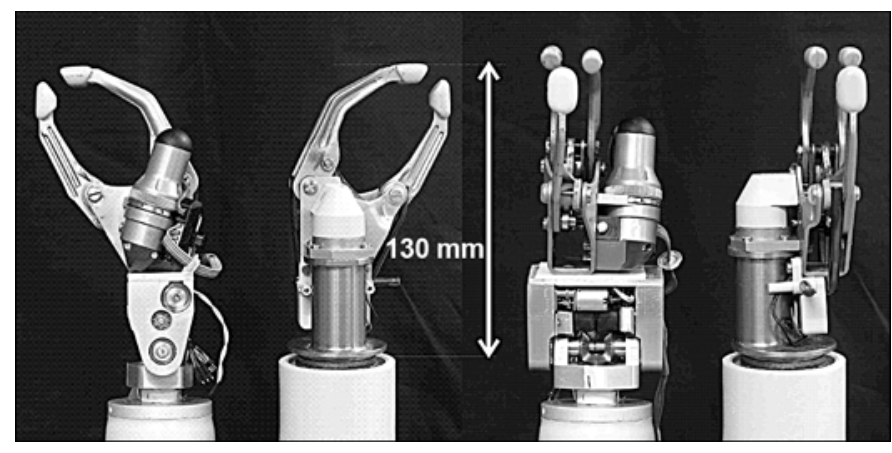

Figure 4.

Second-generation two-axis wrist with Otto Bock transcarpal hand (Otto Bock HealthCare; Duderstadt, Germany) mounted on distal end for comparison with conventional size 7 3/4 hand. Pronation axes are aligned. 
to be achieved and could direct the wrist as he chose. Future quantitative analysis has been planned, which will include a comparative study between the user's conventional myoelectric device and the newer device.

\section{DISCUSSION}

This design shows that combining the output from two drivetrains can provide motion in one axis or two different functions from a unit compact enough to be a practical prosthesis. The wrist's role in positioning the hand to perform tasks is fundamental to reducing compensatory motions in the residual limb. Indeed, direct evidence suggests that a three-DOF wrist can compensate for a hand with limited functional range [2]. Compact wrist systems that can be controlled simply and easily should be pursued to achieve a significant advance in upper-limb prosthesis function. This design, coupled with a flexible pattern-recognition control scheme, may provide a much more functional prosthetic solution.

The performance of the wrist requires some improvements before a truly practical device is created. The wrist is currently fast enough to be practical, the pronation speed being comparable with the only commercially available active wrist, the Otto Bock, which rotates at $80{ }^{\circ} \mathrm{s}^{-1}$ (13.5 revolutions/min). The joints' torques are currently insufficient for general use. Additionally, the mass of the wrist needs to be kept to the current level (200 g) or reduced further. However, it can be backdriven easily, so it cannot be used in the field at the present time.

\section{CONCLUSIONS}

It was demonstrated that a wrist design that possessed two DOFs could be constructed and controlled by a person with limb loss using pattern-recognition techniques on the EMG signals from the forearm. A major manufacturer (RSL Steeper; Leeds, United Kingdom) has announced that they intend to market a two-axis wrist in the near future, although the specifications and how they will be controlled are unknown at the time of writing. The best method to control additional DOFs remains unsolved. If conventional switching of the axes is adopted, then the time taken for a user to activate a DOF will be sufficiently long that they are more likely to use other motions to compensate, obviating the purpose of the new mechanism [32]. Unless the device can be employed quickly and with low mental effort, the only users who are likely to benefit from active wrists will be those with multiple impairments and a greater need for function. Pattern-recognition control may be a viable solution for this problem.

\section{ACKNOWLEDGMENTS}

\section{Author Contributions:}

Study concept and design: P. J. Kyberd, E. Scheme, C. MacPhail, G. Bush.

Mechanical design: E. D. Lemaire, L. Goudreau, M. Brookeshaw. Acquisition of data: P. J. Kyberd, E. Scheme, C. MacPhail, G. Bush. Analysis and interpretation of data: P. J. Kyberd, E. Scheme.

Drafting of manuscript: P. J. Kyberd, E. D. Lemaire, E. Scheme. Critical revision of manuscript for important intellectual content: P. J. Kyberd, E. D. Lemaire, E. Scheme, G. Bush.

Obtained funding: P. J. Kyberd.

Administrative, technical, or material support: P. J. Kyberd, E. D. Lemaire, L. Goudreau, G. Bush.

Study supervision: P. J. Kyberd, E. D. Lemaire, E. Scheme, C. MacPhail, G. Bush.

Financial Disclosures: The authors have declared that no competing interests exist.

Funding/Support: This material was based on work supported by the Canada Chairs Program, the Natural Sciences and Engineering Research Council of Canada (grant 312094-05), and the Atlantic Canada Opportunities Agency (grant 190533).

Additional Contributions: The authors would like to thank the European Union, the Telematics Program, the Canadian Foundation for Innovation, The Ottawa Hospital Rehabilitation Centre, and the Natural Sciences and Engineering Research Council of Canada. They also thank Dr. Harold Sears and Motion Control, Inc, for the loan of the transcarpal hand. Ms. MacPhail is now with the Prosthetic and Orthotic Program, George Brown College, Toronto, Canada.

Institutional Review: This study was approved by the local institutional review board (REB application 2009-055).

Participant Follow-Up: The participant is a long-term volunteer with the program and already aware of the article in process.

Disclaimer: The authors alone are responsible for the content and writing of this article.

\section{REFERENCES}

1. MacPhee B. Examining the prosthetic function and body behaviour of prosthesis users performing Activities of Daily Living [master's thesis]. [Fredericton (Canada)]: University of New Brunswick; 2007.

2. Zinck A. The investigation of compensatory movements in prosthesis users and the design of a novel wrist [master's 
thesis]. [Fredericton (Canada)]: University of New Brunswick; 2008.

3. Sears HH, Iversen E, Archer S, Jacobs T. Wrist innovations to improve function of electric terminal devices. Proceedings of the MEC '08 Measuring Success in Upper Limb Prosthetics; 2008 Aug 13-15; Fredericton, Canada. p. 179-82.

4. Almström C. An electronic control system for a prosthetic hand with six degrees of freedom. Gothenburg (Sweden): Research Laboratory of Medical Electronics, Chalmers University of Technology; 1977. Report No.: 1:77.

5. Berntsson LO, Wiklund P, Almström C, Herberts P, Jönsson LS. [Control of multi-functional hand prosthesis for below elbow amputees by means of identification of EMG signal patterns]. Proceedings of the Annual General Meeting of the Swedish Society of Medicine; 1994; Stockholm, Sweden. Swedish.

6. Almström C, Herberts P, Körner L. Experience with Swedish multifunctional prosthetic hands controlled by pattern recognition of multiple myoelectric signals. Int Orthop. 1981;5(1): 15-21. [PMID: 7275404]

7. Porter LA, Lesley S. An ergonomic one-handed wheelchair. London (UK): Springer; 2002. p. 257-66.

8. Kidd PS, MoCoy C, Steenbergen L. Repetitive strain injuries in youths. J Am Acad Nurse Pract. 2000;12(10):413-26. [PMID: 11930440] DOI:10.1111/j.1745-7599.2000.tb00147.x

9. Jones LE, Davidson JH. Save that arm: A study of problems in the remaining arm of unilateral upper limb amputees. Prosthet Orthot Int. 1999;23(1):55-58. [PMID: 10355644]

10. Fact sheet-Revolutionizing prosthetics program. Arlington (VA): Defense Advanced Research Projects Agency; 2008.

11. Newman P. Multi-articulating hands and fingers-Technical strategies for improved function and myoelectric uptake. Proceedings of the MEC '08 Measuring Success in Upper Limb Prosthetics; 2008 Aug 13-15; Fredericton, Canada. p. 183.

12. Gow D, Marriot B, Douglas WB. A modular prosthetic system for the upper-limb. Proceedings of the International Society of Prosthetics and Orthotics Annual Scientific Meeting; 1989 Apr 12-14; Nottingham, UK.

13. Schulz S. Introducing a new multiarticulating myoelectric hand system. Proceedings of the 13th World Congress of the International Society of Prosthetics and Orthotics; 2010 May 10-15; Leipzig, Germany. p. 598-99.

14. Mell AG, Childress BL, Hughes RE. The effect of wearing a wrist splint on shoulder kinematics during object manipulation. Arch Phys Med Rehabil. 2005;86(8):1661-64.

[PMID: 16084823]

DOI:10.1016/j.apmr.2005.02.008

15. Gillen G, Goldberg R, Muller S, Strauss J. The effect of wrist position on upper extremity function while wearing a wrist immobilising splint. J Prosthet Orthot. 2008;20(1):19-23. DOI:10.1097/JPO.0b013e31815f013f

16. Bertels T, Schmalz T, Ludwigs E. Objectifying of functional advantages offered by wrist flexion. J Prosthet Orthot. 2009;21(2):74-78. DOI:10.1097/JPO.0b013e3181a10f46

17. Kyberd PJ, Poulton AS, Sandsjö L, Jönsson S, Jones B, Gow D. The ToMPAW modular prosthesis: A platform for research in upper-limb prosthetics. J Prosthet Orthot. 2007; 19(1):12-21. DOI:10.1097/JPO.0b013e31802d46f8

18. Kyberd PJ, Chappell PH. The Southampton Hand: An intelligent myoelectric prosthesis. J Rehabil Res Dev. 1994; 31(1):326-34. [PMID: 7869280]

19. Poulton AS, Kyberd PJ, Gow D. Progress of a modular prosthetic arm. London (UK): Springer; 2002. p. 193-200.

20. De Laurentis KJ, Phillips S. Design of a powered two-DOF prosthetic wrist. Proceedings of the 12th World Congress of the International Society of Prosthetics and Orthotics; 2007 Jul 29-Aug 3; Vancouver, Canada. p. 524.

21. Plettenburg DH. The Wilmer 2-DOF Wrist Prosthesis for toddlers. Proceedings of the 12th World Congress of the International Society of Prosthetics and Orthotics; 2007 Jul 29-Aug 3; Vancouver, Canada. p. 522.

22. Dietl H. Prostheses control based on TMR: A case study. Proceedings of the MEC '08 Measuring Success in Upper Limb Prosthetics; 2008 Aug 13-15; Fredericton, Canada. p. 166-70.

23. Pazul K. Controller area network (CAN) basics. Chandler (AZ): Microchip Technology, Inc; 2002.

24. Herberts P, Almström C, Kadefors R, Lawrence PD. Hand prosthesis control via myoelectric patterns. Acta Orthopaedica Scand. 1973;44(4):389-409. [PMID: 4771275] DOI:10.3109/17453677308989075

25. Zhou P, Lowery MM, Englehart KB, Huang H, Li G, Hargrove L, Dewald JP, Kuiken TA. Decoding a new neural machine interface for control of artificial limbs. 2007; 98(5):2974-82. [PMID: 17728391]

26. Kyberd P, Scheme E, Lemaire E, Goudreau L, Brookeshaw M, MacPhail C. A two degree of freedom powered prosthetic wrist controlled using pattern recognition of forearm EMG. Proceedings of the TIPS '09 Trent International Prosthetics Symposium; 2009 May 17-21; Loughborough, UK. Glasgow (UK): ISPO; 2009.

27. Hargrove L, Losier Y, Lock B, Englehart K, Hudgins B. A real-time pattern recognition based myoelectric control usability study implemented in a virtual environment. Proceedings of the 29th Annual International Conference of the IEEE Engineering in Medicine and Biology Society; 2007 Aug 22-26; Lyon, France. Los Alamitos (CA): IEEE; 2007.

28. Kuiken TA, Li G, Lock BA, Lipschutz RD, Miller LA, Stubblefield KA, Englehart KB. Targeted muscle reinnervation for real-time myoelectric control of multifunctional artificial arms. JAMA. 2009;301(6):619-28. 
[PMID: 19211469]

DOI:10.1001/jama.2009.116

29. Bishop W, Armiger R, Burck J, Bridges M, Hauschild M, Englehart K, Scheme E, Vogelstein RJ, Beaty J, Harshbarger S. A real-time virtual integration environment for the design and development of neural prosthetic systems. Conf Proc IEEE Eng Med Biol Soc. 2008;2008:615-19. [PMID: 19162731]

30. Scheme E, Englehart K. A flexible user interface for rapid prototyping of advanced real-time myoelectric control schemes. Proceedings of the MEC '08 Measuring Success in Upper Limb Prosthetics; 2008 Aug 13-15; Fredericton, Canada. p. 150-55.

31. Atkins D, Heard DC, Donovan DH. Epidemiologic overview of individuals with upper-limb loss and their reported research priorities. J Prosthet Orthot. 1996;8(1):2-11. DOI:10.1097/00008526-199600810-00003

32. Kyberd PJ, Murgia A, Gasson M, Tjerks T, Metcalf C, Chappell PH, Warwick K, Lawson SE, Barnhill T. Case studies to demonstrate the range of application of the Southampton Hand Assessment Procedure. Br J Occup Ther. 2009;72(5):212-18.

Submitted for publication August 16, 2010. Accepted in revised form December 14, 2010.

This article and any supplementary material should be cited as follows:

Kyberd PJ, Lemaire ED, Scheme E, MacPhail C, Goudreau L, Bush G, Brookeshaw M. Two-degree-of-freedom powered prosthetic wrist. J Rehabil Res Dev. 2011;48(6): 609-18.

DOI:10.1682/JRRD.2010.07.0137

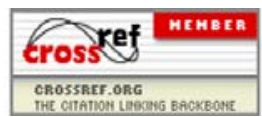


